\title{
La Divina parodia. Un'analisi socioculturale di Dante. La Divina Commedia a fumetti di Marcello Toninelli ${ }^{\mathrm{I}}$
}

\author{
Mario Tirino \\ Università di Salerno \\ mario.tirino@gmail.com \\ Lorenzo Di Paola \\ Università di Salerno \\ lorenzodipaola85@gmail.com
}

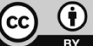

\section{Riassunto}

La forza dell'immaginario nato dalla Divina Commedia ha attraversato secoli e media dando vita a una vasta ed eterogenea selva di prodotti figli dell'eredità dantesca. Il fumetto italiano si è confrontato più volte con il poema "sacro", ma l'unica trasposizione integrale dell'opera è Dante. La Divina Commedia a fumetti di Marcello Toninelli. Questa traduzione fumettistica lascia emergere con ironia il corpo a corpo con la fonte; dinamiche e meccanismi della narrazione poetica vengono parodiati e trasferiti nel linguaggio dei comics, dando vita a una macchina narrativa per immagini in cui, tra innovazione e imitazione, è anche possibile ritrovare l'eco della lunga e fortunata tradizione figurativa del poema.

Parole chiave: Dante; Toninelli; Commedia; parodia; intermedialità.

\begin{abstract}
The power of the imaginary, fueled by Dante's Divina Commedia, has crossed centuries and the media, giving rise to a vast and heterogeneous collection of products of Dante's legacy. The authors of Italian comics have been confronted several times with the "sacred" poem, but the only complete transposition of the work is Dante. La Divina Commedia a fumetti by Marcello Toninelli. This comic book translation has an ironic relationship with the source; dynamics and mechanisms of poetic narration are parodied and transferred into the language of comics. Toninelli creates a narrative machine for images in which, between innovation and imitation, it is also possible to re-discover the echo of the long and successful figurative tradition of the poem.
\end{abstract}

Keywords: Dante; Toninelli; Divine Comedy; parody; intermediality.

I. I due autori hanno concepito e progettato congiuntamente il paper. Mario Tirino ha scritto i paragrafi 3 e 4, Lorenzo Di Paola i paragrafi i, 2 e 5. 


\section{i. Il Dante di Toninelli: \\ UNA STORIA EDITORIALE TRA EVOLUZIONE DEL CONSUMO \\ E TRASFORMAZIONI DELL'INDUSTRIA CULTURALE}

I mondo costruito e rappresentato da Dante nella Commedia ha ispirato 1 per secoli schiere di artisti che, spinti dalle infinite suggestioni nate dalla lettura del "poema sacro cui ha posto mano cielo e terra", si sono confrontati con l'opera dando vita a creazioni originali capaci di correre sui dispositivi più disparati e di intrecciare varie arti. Dall'illustrazione al cinema, dal fumetto ai videogame, non c'è medium che non abbia provato in qualche modo a ri-attivare il discorso, o almeno parte del discorso, dantesco. Grande fortuna hanno avuto le traduzioni visive del poema. L'attività di copia della Commedia è stata fin da subito molto intensa e a breve distanza dalla morte di Dante si cominciano già a registrare i primi esempi della tradizione figurata dell'opera (Pasut 2006). Una tradizione immensa, continuata nell'epoca della stampa con edizioni illustrate da xilografie e giunta fino a oggi passando per tecniche e artisti diversi: soprattutto Gustave Doré (Amendola, Tirino 20I6), ma anche Salvador Dalì, Renato Guttuso, fino alle illustrazioni di Moebius e Lorenzo Mattotti, solo per fare qualche esempio della sterminata produzione. La lista si allunga vertiginosamente se proviamo ad aggiungere sculture, dipinti, film e fumetti che provano a tradurre l'opera in un altro linguaggio. Sin dall'epoca della tradizione manoscritta è evidente che le miniature, le illustrazioni, fungono da vere e proprie glosse visive, capaci di orientare verso determinati modelli interpretativi il senso dell'opera. Ogni trasposizione della Commedia porta con sé una valutazione critica dell'artista che inevitabilmente privilegia prospettive vicine alla propria sensibilità culturale. Le miniature non sono solo un mero ornamento estetico, ma veri e propri corredi esegetico-interpretativi, strettamente legati all'idea della Commedia di colui che ha guidato la mano dell'artista:

Che visualizzino le tappe di un viaggio assumendo modelli figurativi dalla tradizione, pertinente, delle Eneidi illustrate, [...] o costruiscano paratesti di forte valenza allegorico-morale [...] o facciano della Commedia una sorta di trattato di casistica morale assumendo il modello librario proprio della tradizione universitaria e in particolare di quella giuridica [...] è da una precisa opzione critica, da una ben determinata "idea di Dante" che ognuno di questi libri nasce, ed è quella medesima "idea" che ognuno di essi trasmette a chi quelle stesse carte si troverà a sfogliare, fortemente orientando la lettura del testo poetico lì registrato (Ricci 20II: 564).

Dunque, anche il fumetto quando prova a confrontarsi con l'opera dell'Alighieri, inevitabilmente, trasmette una ben determinata idea della Commedia e sono molti i fumettisti che si sono cimentati in questa impresa, nonostante la complessità e la distanza del testo che, in veste di poema, dà forma all'esperienza ultraterrena vissuta dall'autore-personaggio Dante, il quale pretende 
che il racconto sia assunto come veridico ed esemplare sul modello delle sacre scritture (Giunta 20I5). In queste pagine, attraverso l'analisi di Dante. La Divina Commedia a fumetti di Marcello Toninelli, proveremo ad esplorare le dinamiche con cui i meccanismi della narrazione poetica si trasferiscono nel linguaggio dei comics e in che modo innovazione e imitazione riescono a mettere in moto una macchina narrativa per immagini in cui è possibile ritrovare l'eco di una lunga e fortunata tradizione figurativa.

Il Dante di Toninelli attraversa varie fasi cruciali della storia del fumetto italiano (Brambilla 2015). L'esordio avviene il ro novembre del 1969 sull'undicesimo numero di Off-Side. Tutto il lato destro della copertina è occupato da un Dante stilizzato e dal suo baloon: "Fatti non foste a legger comics bruti, ma per seguir storielle di valenza!" (Fig. I). Sono anni, questi, decisivi per il fumetto italiano e la rivista, con i suoi due anni di vita e i diciassette numeri pubblicati, ha dato sicuramente un importante contributo al radicale rinnovamento del medium. Off-Side nasce sulla scia di Linus (1965), la prima rivista "adulta" nel mondo dei comics italiano, grazie a cui il fumetto diventa una faccenda seria (storica la conversazione a tre sul primo numero tra Umberto Eco, Elio Vittorini e Oreste del Buono). Infatti, vi trovano spazio preziose analisi del medium e viene presentato il meglio della produzione statunitense e italiana, legando il fumetto alla vita sociale e politica del Paese: "questa operazione è importante perché sottrae il fumetto al ghetto in cui la cultura italiana l'ha tradizionalmente rinchiuso, e prepara una generazione di autori e lettori destinata a vivere un nuovo tipo di relazione con la letteratura per immagini” (Barbieri 2009: I22). Per distinguersi da Linus, Off-Side opta per un formato tabloid di 32 pagine in bicromia dal costo contenuto, consente la riscoperta dei classici americani e la scoperta di grandi autori italiani, tra cui Guido Crepax con la sua L'astronave pirata e le Sturmtruppen di Bonvi, e ospita rubriche culturali e politiche. Il Dante di Toninelli in queste pagine possiede un tratto ancora incerto, in divenire, ma il formato con cui presenta la sua Commedia è già quello definitivo, quello tipico della striscia formata da due, tre vignette con battuta finale; domina il bianco e nero, unica eccezione la tunica di Dante che si adatta al colore, sempre diverso, della rivista in bicromia. Con la chiusura di Off-Side, alcune strisce della Commedia compaiono su un'altra sfortunata rivista, Undercomics, nel 1972, per approdare poi nel 1985 su Fox Trot e nel 1989 su Fumo di China. In questo viaggio fra riviste il tratto di Toninelli acquista maggiore stabilità, evolve verso la linea chiara e i paesaggi di sfondo si fanno più definiti. È solo quando don Tommaso Mastrandrea, direttore de Il Giornalino, settimanale delle edizioni San Paolo, decide di ospitare Toninelli nelle pagine del periodico cattolico, che le tre cantiche vengono regolarmente pubblicate, dal 1994 al 1998. Ovviamente la pubblicazione su un settimanale cattolico per ragazzi richiede alcune trasformazioni in linea con il programma 
ludico-didattico della rivista. Nelle strisce, allora, compaiono colori e vestiti per le anime prima ignude, e la componente didattica (sempre smorzata dalla consueta ironia di Toninelli) acquista maggior rilievo anche per consentire ai giovani lettori di comprendere i complessi riferimenti storici-biografici e teologici (Fig. 2). Nel 1994 una versione in tre volumi, uno per cantica, viene pubblicata da Cartoon Club.

Nel 2015 la casa editrice Shockdom raccoglie in volume tutte le strisce in una edizione che contiene le tre cantiche e La vita di Dante a fumetti. Come abbiamo visto, questa Commedia a fumetti è giunta a noi, attraverso gli anni e le riviste, con molti cambiamenti, ed è molto interessante notare come sia una casa editrice specializzata nel settore del webcomic a ripubblicare l'opera di Toninelli. La scelta della Shockdom dice molto sulla produttività di un formato, quello della striscia, che da più di un secolo accompagna le vite dei lettori. Il fumetto, oggi, è sottoposto a "cambiamenti imprevisti, a tensioni che da un lato ne stanno mutando gli assetti produttivi e le modalità di consumo, e dall'altro restano sospese, in attesa di un futuro assestamento" (Frezza 2017: 9) ed è innegabile che si sia spezzato quello speciale rapporto tra fumetti e daily strip dei quotidiani che, per molto tempo, specialmente negli Stati Uniti, ha fatto da motore alla produzione dei comics, cementando e sostenendo relazioni tra autori, syndacates e pubblici di lettori. La striscia, però, non muore con i quotidiani e ritrova linfa vitale nel web, dove diventa uno dei formati privilegiati della comunicazione digitale, dando vita a nuove forme di produzione e di distribuzione. La Commedia di Toninelli è testimone e oggetto di tutte queste trasformazioni del medium e del suo cambiamento da mezzo di comunicazione popolare a medium di nicchia, riservato ad un pubblico ben selezionato; il suo Dante ha assistito alla morte delle riviste d'autore e all'affermarsi del graphic novel, formato stabilizzato nel mercato attuale, in cui sono ripubblicate anche vecchie opere raccolte in volume.

Il web e il digitale rivoluzionano ovviamente il fumetto. Oggi si legge sempre meno su carta e sempre più su varie tipologie di schermi, ma la striscia continua a popolare e affollare la selva dei comics e a nutrire un'editoria via via più specializzata e attenta ai rapporti, sempre più personali, fra lettore $\mathrm{e}$ opera. La pubblicazione di Dante. La Divina Commedia a fumetti da parte della Shockdom può inoltre essere letta come una delle tante operazioni editoriali volte alla conservazione di opere considerate classici del fumetto.

\section{L'esteTiCA TONINELLIANA:} STRISCIA, LINEA CHIARA, CARICATURA

C’è da dire che la Commedia di Dante ha offerto numerosissimi casi di riscritture parodiche, soprattutto in Italia, a partire da Linferno di Topolino scritta da 
Guido Martina e disegnata da Angelo Bioletto (pubblicata sui numeri 7-I2 del periodico Topolino, tra ottobre 1949 e marzo 1950), in cui assistiamo anche a uno spassoso travestimento comico della terza rima dantesca. Toninelli, però, è uno dei pochi autori a portare a termine una trasposizione fedele dell'opera, parodiando tutto il poema, canto per canto. A differenza di altre parodie, l'autore decide qui di affidarsi solo alla forza dialogica dei balloon senza ricorrere all'ausilio di versi o di didascalie, la battuta finale della striscia e il rapporto da tipica coppia comica tra un Dante inadeguato e scanzonato e un Virgilio bonario e sfortunato fanno poi da carburante a buona parte della narrazione. Pochi gruppi di terzine offrono materiale sufficiente alla realizzazione di una striscia. Toninelli mette a frutto la sua esperienza, maturata all'interno dell'industria culturale del fumetto, come disegnatore, sceneggiatore, redattore ed editore, e trasforma con sapienza le occasioni offerte dalla scrittura di Dante in un prodotto visivo e seriale. La Commedia, lungi dall'essere vista come una "sceneggiatura di ferro", è usata sornionamente come una sorta di "scaletta", ovvero come uno "strumento utile e duttile a contemplare una molteplicità incalcolata di possibilità di definizione del prodotto" (Frezza 1987: 9) ed è proprio questa duttilità a permettere la trasformazione del testo dantesco in miniera inesauribile di gag. Toninelli si dimostra consapevole della distanza esistente tra la scrittura di un testo letterario, che "definisce lo spazio poetico e narrativo in cui possono includersi [...] immagine evocate", e la scrittura di una sceneggiatura che predefinisce "elementi che saranno tecnicamente finiti tramite ulteriori processi generativi” (Frezza 1987: 6). Trasforma e ri-configura questa distanza, agisce sulla materia del testo rimodellando spazi e tempi nel codice del fumetto in un gioco continuo tra fedeltà e infedeltà, ripetizione e innovazione. Quest'opera poi si offre sicuramente come un ottimo esempio della capacità della scuola italiana del fumetto di riuscire a fare propria la lezione dei grandi maestri della daily strip statunitense e internazionale. Da Linus in poi, in Italia, vengono pubblicate tantissime strisce e Toninelli sembra introiettarne perfettamente regole e ritmi. I Peanuts, Mafalda, Lucky Luke, Beetle Bailey, The Wizard of Id, i fumetti di Mordillo, e molti altri concorrono alla formazione del peculiare universo narrativo di Toninelli. A quest'eredità va aggiunta una personalissima linea chiara, frutto di una lunga evoluzione stilistica, fatta di linee essenziali e precise. L'assenza di modulazione e di tratteggi fa sì che ogni forma venga trattata alla stessa maniera, una scelta di semplicità tesa a rendere la narrazione grafica più immediata e diretta possibile. Il suo è un segno che cerca di contrapporre la chiarezza della linea e la semplicità del disegno all'enorme complessità dell'opera dantesca. Sono tutti strumenti che, insieme all'uso di balloon quadrangolari, richiamano alla mente la bande dessinée della scuola belga. Toninelli, insomma, mette a frutto, in maniera semplice e naturale, la lunghissima ed eterogenea tradizione del 
fumetto "comico-avventuroso". Non a caso gioca un ruolo importantissimo la caricatura, campo in cui Toninelli si dimostra un maestro grazie alla sua capacità di tradurre in pochi tratti un universo gigantesco come quello della Divina Commedia. Grazie alla caricatura, la comunicazione si fa più coinvolgente, più appassionante e la velocità e l'essenzialità di questo strumento vanno perfettamente a braccetto con il formato della striscia. "La caricatura è lo strumento per evidenziare le caratteristiche di ciascun personaggio: caricandone alcuni aspetti li si rende tipici e facilmente riconoscibili (...) la caricatura è adatta all'umorismo perché è in grado di dirci molte più cose in un colpo solo di quanto non lo sia l'immagine realistica" (Barbieri 1990: 70). Sicuramente Toninelli, in questa riduzione, è aiutato anche dalla famosa e lunga tradizione grafica, di cui abbiamo già accennato, della Commedia che ha trasformato molti luoghi del poema in momenti iconici e facilmente riconoscibili, a partire dalla rappresentazione dello stesso Dante.

\section{Cortocircuiti: Dante e l'immaginario pop}

In maniera giocosa, ma sempre rispettosa del testo, nel Dante di Toninelli viene modificato il "livello di dignità" dell'opera (Genette 1997), inserendo una serie di anacronismi, rinvii e citazioni attinti dalla cultura pop. Occorre tuttavia necessariamente collocare l'opera di Toninelli in due cornici culturali più ampie: da un lato, l'esperienza specifica della parodia disneyana; dall'altro, il sistema dei media e dei consumi dell'Italia degli anni Ottanta e Novanta.

La parodia è il genere "attraverso cui l'universo disneyano ha assunto dentro di sé l'intero campo dei classici della letteratura, l'immaginario cinematografico e televisivo, le tecniche dell'informazione e dello spettacolo sul piccolo schermo" (Frezza 1987: 46). Nelle sceneggiature di autori come Guido Martina, Luciano Bottaro, Giovan Battista Carpi, Romano Scarpa, le storie a fumetti di paperi e topi, dunque, si lasciano compenetrare da mitologie nutrite dalla cultura di massa. La comunicazione di massa si rivela uno dei luoghi di mitopoiesi più fervidi della contemporaneità (Morin 20I7), talvolta eccedendo i controlli, i limiti e le censure dei saperi istituzionali (Abruzzese 1973, 2007), talvolta - come nel caso dei fumetti Disney - vivificando le fonti letterarie tradizionali e, in tal modo, creando canali di dialogo anche con le istituzioni delegate alla trasmissione del sapere: "La parodia dei classici letterari, adattati all'universo dei paperi e dei topi, si è innestata con fertili risultati sul corpo della cultura scolastica italiana del dopoguerra, presentandosi come uno strumento di divulgazione del sapere letterario e conquistandosi un mercato e un target di lettori (bambini-adolescenti)" (Frezza 1987: 46-47). In sintesi, è con il modello della parodia dei grandi classici della letteratura, sviluppato dai fumetti Disney italiani, che la macchina per immagini di Toninelli deve 
necessariamente confrontarsi (il tema della parodia a fumetti Disney è trattato in maniera più approfondita nel saggio di Daniela Pietrini, che compare in questo stesso numero della rivista).

Anche per quanto concerne la relazione tra il Dante del fumettista senese e il sistema dei media, non si può fare a meno di ribadire, innanzitutto, che, sebbene dentro i limiti di un'industria culturale mai pienamente sviluppata come quella italiana (Colombo 1998), già la parodia disneyana, come scritto in precedenza, aveva aperto la strada a una rimediazione delle culture letterarie e di quelle audiovisive, nello spazio rappresentativo e simbolico di strisce, tavole e vignette.

Rispetto alle due storie disneyane dedicate all'Inferno dantesco, il citato L'Inferno di Topolino, e L'Inferno di Paperino, scritta e disegnata da Giulio Chierchini (pubblicata sul numero I654 di Topolino nell'agosto 1987), il Dante di Toninelli "è decisamente più fedele all'archetipo (...), di cui segue puntualmente la trama e lo schema delle punizioni e riprende protagonisti, personaggi e ambientazioni infernali" (Benucci 2018: I09).

Su questo schema narrativo, che, come già evidenziato, ha alimentato negli anni una tradizione figurativa imponente, si inserisce però una fittissima rete di rimandi, prelievi, suggestioni intermediali, da cui il fumettista senese attinge a piene mani per varie finalità. Intanto, va rilevato preliminarmente che il milieu culturale dentro cui affondano le radici dell'umorismo di Toninelli è evidentemente la cultura italiana popolare degli anni Ottanta e Novanta. L'immaginario della comunicazione di massa viene letteralmente saccheggiato dal disegnatore toscano e inserito, con effetti comici, farseschi o umoristici, nella tessitura della narrazione dantesca: dal cinema alla televisione, dalla pubblicità alle fiabe popolari, dalla letteratura al costume e allo sport, nessun materiale culturale popolare viene trascurato.

Sin da subito, il cinema si rivela tra le fonti più amate da Toninelli per sovvertire l'ordine spaziotemporale della Commedia con spericolati anacronismi. Gli esempi sono numerosi e diversificati. Virgilio, che si presenta come "colui che ha narrato le gesta dell'eroe fuggito dalla città più famosa di tutti i tempi", viene scambiato da Dante per Jena Plissken, il leggendario antieroe interpretato da Kurt Russell in 1997: Fuga da New York (198I) e Fuga da Los Angeles (1996), entrambi diretti da John Carpenter (If. I, Toninelli 20I5: II, d'ora in poi solo $\mathrm{T}$ ). Celestino $\mathrm{V}$, interrogato da Dante sul perché abbia rinunciato al soglio pontificio aprendo la strada agli intrighi di Bonifacio VIII, risponde, stringendo in mano una testa di cavallo mozzata, con una celebre frase de Il Padrino (1972, di Francis Ford Coppola): "Mi ha fatto un'offerta che non potevo rifiutare" (If. III, I4). Le orribili amputazioni, che devono subìre scismatici e seminatori di discordia, vengono "somministrate" dagli artigli di Freddy Krueger, il personaggio, interpretato quasi sempre da Robert Englund, 
protagonista della longeva saga horror Nightmare (nove film dal I984 al 20I0) (If. XXVIII, 79). Tra i falsari di parole, divorati da orribili febbri, se ne distingue uno che ha l'aspetto di John Travolta ed è infatti squassato dalla particolare febbre del "sabato sera", in omaggio al cult movie La febbre del sabato sera (1977, di John Badham) (If. XXX, 84). I giganti, che sfidarono gli dei e pertanto sono incatenati al pozzo che contiene l'ultima parte di Inferno, sono disegnati come musicisti che usano una scimmia particolare per raccogliere le offerte del pubblico: si tratta di un'autentica icona dell'immaginario contemporaneo (Frezza 2006, Abruzzese 2007), ovvero quel King Kong, che appare sugli schermi per la prima volta nell'omonimo film del 1933 per passare poi attraverso numerosi remake e reinterpretazioni (If. XXXI, 85). Un altro caposaldo del cinema degli anni Ottanta viene chiamato in causa quando compare la squadra di diavoli acchiappa-anime, incaricati di togliere un'anima dal corpo di un demonio e mutuati sul modello degli acchiappa-fantasmi protagonisti della saga Ghostbusters (tre film dal I984 al 20I6, più serie animate e videogame) (If. XXXIII, 89). Gli angeli, deputati a scacciare il serpente dal Purgatorio, sostituiscono le vecchie spade lucenti con un nuovo modello di spade laser, il cui utilizzo è abbinato al grido propiziatorio "Che la forza sia con te", in un delizioso tributo alla saga Star Wars creata da George Lucas (Pg. VIII, IO6). Toninelli omaggia anche il classico Mezzogiorno di fuoco (1952, di Fred Zinnemann), attraverso l'escamotage di un angelo appassionato di western che, per cancellare una delle sette "p" dalla fronte di Dante, utilizza un proiettile di gomma invece di un colpo d'ali (Pg. XII, II4). Probabilmente, però, la contaminazione più divertente tra strisce e culture filmiche, avviene quando il Sommo Poeta, interrogato sul tema del De Trinitate del teologo Riccardo di San Vittore, afferma che il testo tratta di film italiani, ovvero Lo chiamavano Trinità (1970) e ...continuavano a chiamarlo Trinità (197I), entrambi diretti da Enzo Barboni e interpretati dalla coppia Bud Spencer - Terence Hill (Pd. X, I63) (Fig. 3). In questo scambio di battute ai limiti dell'assurdo, il fumettista toscano fa collidere in maniera spettacolare cultura alta e cultura bassa, i trattati teologici e gli spaghetti western, la meditazione filosofica e la commedia popolare. Infine, ad un San Pietro irritato per la trasformazione dei pastori della Chiesa in "lupi avidi e voraci", e che si chiede chi difenderà il gregge dei fedeli, risponde un Parrocop, versione "ecclesiale" del Robocop, poliziottorobot dell'omonimo media franchise fantascientifico ( $P d$. XXVIII, I95).

Il cinema si insinua nella parodia toninelliana anche come forma di motto, quando il cugino del padre di Dante, Geri del Bello, parafrasa il famoso slogan mutuato dal film Rambo II - La vendetta (1985, di George Pan Cosmatos) (If. XXIX, 80), seconda di quattro pellicole dedicate al veterano del Vietnam interpretato da Sylvester Stallone; oppure quando Beatrice pronuncia la formula per il rientro nell'alto dei cieli, ovvero "telefono-casa", come quella dell'alieno 
E.T. nel capolavoro E. T. - L'extra-terrestre (1982, di Steven Spielberg) (Pg. XXXIII, I49).

Particolarmente riuscita ci sembra la soluzione adottata dal fumettista toscano per rappresentare le vite dei beati: si tratta, come preannuncia Beatrice a Dante, di brevi trailer, corredati da giochi linguistici per i titoli (Pd. XVIII, I80-I82). In tal modo, la storia di Giuda Maccabeo è raccontata nel film $I l$ cacciato re di Michael Virtuino (da Il cacciatore, 1978, di Michael Cimino) (Fig. 4); la vicenda di Carlo Magno è narrata in Indovina chi viene al cenone di Stanley Virtuamer (da Indovina chi viene a cena?, 1967, di Stanley Kramer); le peripezie di Orlando vengono rielaborate ne La notte dei mori vincenti di George Virtuero (da La notte dei morti viventi, I968, di George Romero); la vita di Goffredo di Buglione diventa il biopic kolossal Ricomincio da re di Massimo Virtuisi (da Ricomincio da tre, 198I, di Massimo Troisi); infine, le gesta di Roberto il Guiscardo sono cantate in Via col Berto (da Via col vento, 1939, di Victor Fleming). Dante apprezza talmente il gioco dei trailer, da proporre egli stesso, qualche pagina dopo, la realizzazione di un trailer sulla storia di Davide e Golia, proponendone pure il titolo Gli uomini preferiscono le fionde (da Gli uomini preferiscono le bionde, I953, di Howard Hawks) (Pd. XIX, I83).

Sporadicamente, è possibile rintracciare anche alcune fugaci citazioni di star contemporanee, come Bruce Willis (If. XXXII, 87), Angelina Jolie (If. XXXIV, 9I) e Brad Pitt (Pd. XX, I85).

Come si può vedere dagli esempi estrapolati, Marcello Toninelli predilige il cinema di genere, i cui topoi visivi e narrativi sono quasi immediatamente riconoscibili e interpretabili dal lettore. Il suo obiettivo resta sempre creare un clima di complicità con chi legge, anche se dal repertorio delle citazioni filmiche sembra che il patrimonio di conoscenze saccheggiato dall'autore sia più facilmente condivisibile con un pubblico di adulti o giovani adulti.

Nel Dante di Toninelli resta lo spazio per una simpatica incursione nell'evoluzione della tecnologia del cinematografo, che rivoluziona persino le abitudini percettive dell'aldilà: invece di apparire direttamente nella mente delle anime come un tempo, le visioni sono ora proiettate, "in stereocolorvision", attraverso una sorta di moderno peep show su una sfera cristallo, in cui, dietro pagamento di un fiorino, è possibile visionare una serie di esempi di ira punita da Dio (Pg. XVII, I22).

Ma è soprattutto la cultura televisiva che modella e permea di sé l'intera operazione intermediale di Marcello Toninelli. L'elenco delle occorrenze è lungo ed eterogeneo. Nel primo cerchio dell'Inferno, mentre Virgilio spiega a Dante che l'unica pena delle anime di virtuosi non battezzati è non poter vedere Dio, una di loro si lamenta del fatto che con il pessimo televisore infernale si riesca appena a vedere Telenorba (If. IV, I6). Nella striscia successiva, Dante viene a conoscenza del fatto che gli unici ad abbandonare il Limbo 
sono stati Adamo, Abele, Noè, Mosè, Abramo e Re Davide, perché "nominati in varie edizioni del Grande Fratello" (If. IV, I6). Gli eroi troiani Ettore ed Enea svelano che il loro piano segreto per mettere in fuga gli assedianti greci consisteva nel donare loro una televisione perennemente sintonizzata su Beautiful, giocando sul fatto che la visione della soap opera americana avrebbe assorbito attenzioni ed energie distogliendo gli spettatori dall'attacco alle città troiane (If. IV, I9). Le telenovelas ricompaiono anche per ironizzare sullo statuto di celebrity degli interpreti: così il sultano d'Egitto Ibn Ayyub Salah $\mathrm{Al}$-Din viene considerato da tutti, non più un feroce guerriero, ma "un uomo saggio, colto e coraggioso" per aver interpretato questa parte in una telenovela trasmessa da Al Jazeera (If. IV, 20). La stessa Cleopatra svela al Sommo Poeta le vere ragioni del suo suicidio: il rinnovo, per l'ennesima stagione, di Pharaon Dynasty (If. V, 26), nome che evoca l'infinita soap opera americana Dynasty, andata in onda dal 198I al 1989 (per un totale di 220 puntate).

Per gli studi di sociologia e mediologia del fumetto, merita una trattazione a parte la costruzione estetica di Toninelli nella sequenza delle "sculture viventi”, raffiguranti esempi di superbia punita. Qui sembra che l'autore senese pensi al fumetto come uno spazio simbolico in cui riflettere sulla medialità contemporanea, ovvero come un medium "baricentrico" nel mediascape, in grado di organizzare, manipolando le proprie convenzioni figurative, la "rimediazione" (Bolter, Grusin 1999) di un medium classico (la scultura) attraverso i codici di un medium contemporaneo (la televisione): in altri termini, la serie di sculture viene organizzata visivamente come un flusso di immagini televisive (Pg. XII, II2-II3) (Fig. 5), come dimostrano la vignetta in cui l'artista scolpisce la classica didascalia utilizzata dalle emittenti per i problemi tecnici (" $\mathrm{Ci}$ scusiamo per l'interruzione. La trasmissione riprenderà appena possibile”), quella con i consigli per gli acquisti ("Pena più leggera con i massi in pietra pomice Senza Sforzo"), quella con il "fan dei pietroni animati della Donna Ragna” (con chiaro omaggio a Spider-man), quella con il riepilogo delle puntate precedenti della "pietranovela" Anche gli sciti piangono (lampante richiamo alla popolarissima telenovela messicana Anche i ricchi piangono, a più riprese trasmessa da differenti network italiani negli anni Ottanta, e parodizzata pure come Anche i santi piangono, in Pd. XXI, I87).

Nella televisione commerciale degli anni Ottanta si affermano inoltre i primi vip del piccolo schermo, che, con la loro compagnia quotidiana, diventano i beniamini di un vasto pubblico, talora segnato da consumi generazionali. L'icona dei teenager Cristina D'Avena, voce di tante sigle dei cartoon trasmessi in Italia in quegli anni, è il bersaglio della satira del fumettista senese: Dante, infatti, la indica come una disgrazia peggiore di ogni guerra a Guido da Montefeltro, ansioso di conoscere il destino della sua Emilia-Romagna (If. XXVII, 78). Uno dei principali programmi della televisione italiana, il 
Maurizio Costanzo Show, è parodiato come "San Bonaventura Show", in cui il conduttore San Bonaventura, accompagnato da un pianista del tutto simile a Franco Bracardi, presenta in successione una serie di beati che sfilano sul palcoscenico proprio come gli ospiti del seguitissimo talk di Costanzo (che pure non viene mai menzionato) (Pd. XIII, I72-I74).

Persino il rapporto tra Dante e l'amata Beatrice viene re-immaginato da Toninelli nella dimensione spettacolare dei media audiovisivi: la donna tanto desiderata appare ora come una lanciatissima soubrette, che si fa chiamare Bea Trix, canta Ti parlerò d'amor (il brano reso celebre da Wanda Osiris che lo incise nel 1945), fa la direttrice artistica dei programmi del Paradiso ed è assorbita da problemi come la gestione dello staff di collaboratori e l'indice di popolarità (Pg. XXX, I42-I43). Dello stesso tenore appare la parodia del quiz La ruota della fortuna, che diventa "La ruota della cultura", condotta da un San Tommaso d'Aquino che fa il verso a Mike Bongiorno, tic e fedeltà allo sponsor compresi (Pd. X, I62-164) (Fig. 6). Toninelli non lesina un omaggio anche alla rivista più amata dai telespettatori italiani, $T$ v, sorrisi e canzoni, trasformata nell'universo fumettistico in "Quassù sorrisi e canzoni". Il quiz show ritorna poche pagine dopo, quando Toninelli trasforma le prove cui è sottoposto l'Alighieri (riconoscere le anime in una schiera ampia a forma di aquila) in un omaggio a Scommettiamo che, tra i più longevi e popolari programmi della televisione italiana ( $P d$. XVIII-XIX, I82-I83). I riti e i miti del mondo televisivo sono oggetto di due simpatici anacronismi: Ulisse rivela l'insofferenza per Penelope che, ogni sera, lo costringeva a guardare la tela da lei tessuta durante l'intera giornata, chiamando questa pratica "tela-visione" (If. XXVI, 77) e Carlo Martello che, appassionato di felini, intitola il concorso di pittura da lui istituito "tela-gatto" ( $P d$. VIII, I58) (con ovvio rimando ai celebri premi della televisione italiana). Lo stesso intermezzo delle previsioni meteo, che, negli anni Ottanta e Novanta, erano esclusivo appannaggio dell'Aeronautica Militare, è oggetto della parodia toninelliana, che ha luogo quando un angelo presenta le "previsioni sul Dante", "a cura dell'ufficio previsiologico dell'angelonautica" ( $P d$. XVII, I77).

Fioriscono, inoltre, i prelievi dagli universi del fumetto e dei cartoni animati. Quando Virgilio presenta a Dante Ptolomeo, compare d'improvviso Eta Beta, il personaggio proveniente dal futuro, creato da Floyd Gottfredson e Bill Walsh per la storia disneyana Mickey Mouse and the man of tomorrow (1947), noto per il difetto di pronuncia che lo porta ad anteporre la lettera "p" ad ogni parola (If. IV, 22). Particolarmente esilarante è l'apparizione degli orsi Yoghi e Bubu, protagonisti di serie televisive animate (dal 1958 al 1992), lungometraggi e videogiochi prodotti dallo studio Hanna-Barbera, che compaiono in qualità di "parenti" di papà Niccolò III, nato Giovanni Gaetano Orsini, collocato da Dante tra i simoniaci dell'ottavo girone infernale (If. XIX, 63) 
(Fig. 7). Altrettanto improvvisa è l'apparizione di Obelix, il gigante panciuto coprotagonista della serie di fumetti e lungometraggi animati Asterix, creata da René Goscinny e Albert Uderzo: l'insaziabile guerriero dei Galli saluta, dal girone dei golosi in cui è collocato da Toninelli, il connazionale Ugo Capeto (Pg. XX, I26). Nella stessa sfera delle citazioni irriverenti possiamo includere la fulminea comparsa di Gatto Silvestro, mentre San Tommaso sta elencando i primi seguaci di San Francesco, tra cui Bernardo di Chiaravalle, Egidio e, appunto, Silvestro: tuttavia il celebre felino, personaggio dei cartoni animati di Looney Tunes e Merrie Melodies, a differenza dell'omonimo frate, afferma di essersi pentito subito della scelta di seguire il francescanesimo, poiché "quel testone pretendeva che trattassimo come nostri fratelli anche i canarini" ( $P d$. XI, I65), notoriamente il pasto agognato da Silvestro, anche se mai consumato per l'agilità di Titti. È rivolto in particolare ai cultori del fumetto l'omaggio al Signor Bonaventura, il personaggio, creato da Sergio Tofano (Sto), che riscosse maggior successo nel fumetto italiano dell'anteguerra e fu trasposto anche in commedie musicali e un lungometraggio: il fumettista toscano dà a San Bonaventura proprio le fattezze della squattrinata creatura a fumetti di Sto (Pd. XII, I69). Un discorso a parte meritano i rinvii all'universo dei supereroi; Superman è chiamato in causa ben tre volte (Achille rivela a Dante che il suo punto debole non è il tallone, ma la kryptonite, scoprendo il petto dove campeggia la "S" del personaggio a fumetti creato da Joe Shuster e Jerry Siegel nel I933, If. V, 26; il Sommo Poeta scambia l'imperatore Manfredi per Superman "che si è dimenticato di indossare il costume", $P g$. III, 98; Beatrice fa indossare a Dante un adesivo con l'iniziale del proprio nome e gli fa alzare il braccio con il pugno chiuso, proprio come Superman, perché "per volare fa più scena", $P d$. I, I53).

Va evidenziato un notevole esercizio di metafumetto, allorché l'Alighieri chiarisce a Griffolino d'Arezzo che, quando parla di "senesi gente vanesia", si riferisce proprio "all'autore di queste strisce, che le firma tutte!" (If. XXX 82) (Fig. 8).

Della cultura fumettistica degli anni Novanta fanno parte anche i manga, anche se all'epoca meno distribuiti e letti di quanto non lo siano oggi. Le parole di San Bonaventura, che rievoca il sogno della madrina di San Domenico di "un fanciullo che aveva una stella in fronte", del quale poi vendette i diritti per una serie a cartoni animati, sono l'occasione per citare il manga Sailor Moon (I99I-I998), creato dalla fumettista Naoko Takeuchi e sviluppatosi come vero e proprio franchise transmediale ( $P d$. XII, 169) (Fig. 9). Non si tratta di una citazione isolata: occhieggiando alla generazione Goldrake, ovvero a quegli adolescenti cresciuti con gli anime trasmessi dalle reti televisive private (Pellitteri 20I2, Teti 20I2), il Dante di Toninelli "vede" in un'anima che vortica furiosamente su se stessa il personaggio di Gigi la trottola, protagonista della 
omonima serie manga ideata da Noboru Rokuda nel 1980 e diventata l'anno successivo un popolarissimo anime ( $P d$. XXI, I86).

Ancora, appaiono dal nulla i tre porcellini, rappresentati secondo lo stile del cartoon Disney tratto dall'omonima fiaba popolare inglese (I tre porcellini, 1933, di Burt Gillett), che contestano San Tommaso, affermando che al feroce lupo di Gubbio avevano pensato già loro, prima di San Francesco (Pd. XI, I66). D'altronde, Toninelli attinge al repertorio delle fiabe popolari, come quando un diavolo paragona Virgilio e Dante al principe azzurro e a Cappuccetto Rosso per il loro abbigliamento (If. VI, 29).

Meno frequente è il ricorso a modelli letterari. Un diavolo con una gamba di legno è ossessionato dalla balena bianca che lo ha privato dell'arto, vicenda che, secondo Dante, potrebbe essere materia di un romanzo, con uno scoperto rinvio a Moby Dick (I85I) di Herman Melville (If. X, 35-36). Alla ricerca di un titolo per l'opera che dovrà raccontare ai vivi il mondo degli inferi, Dante partorisce un titolo che è un omaggio a uno dei tanti eccezionali romanzi d'avventura di Jules Verne: Viaggio al centro della Terra (I864) (If. XXXIV, 9I). In un altro momento salta fuori, quasi d'improvviso, Pinocchio che scorge, dietro la figura allegorica della volpe che simboleggia "l'eresia che minaccia la religione dall'interno", la coda di un gatto, e che informa i pellegrini che "quei due non si separano mai" (Pg. XXXII, I46).

Altrettanto limitati risultano i riferimenti alla musica, più che altro riservati a eventi altamente spettacolari e nazionalpopolari, come il Festival di Sanremo (Pg. II, 97) e il Cantagiro ( $P d$. XXIII, 190). Poco più di un gioco di parole è la risposta del Sommo Poeta al suo maestro, quando questi gli chiede, a proposito dei superbi, come avesse fatto a non accorgersi che gli enormi massi rotolanti erano mossi da uomini: Dante afferma di aver pensato a "una tournée dei Rolling Stones" (Pg. X, IIO).

Protagonista di uno dei primi media franchise di successo globale, anche l'eroina dei videogames Lara Croft viene citata da Pantasilea, la regina delle Amazzoni assassinata da Achille, come la donna che, nel futuro, sarà capace di "tenere testa agli uomini" (If. IV, 20). La successiva apparizione dei game concerne un'interessante pratica di comunicazione intermediale congegnata da Toninelli: qui Gesù è invitato dalla Madonna a spegnere il Dio-Game con cui sta giocando, mentre sul display compaiono le immagini di Dante e Virgilio che proseguono il loro cammino (Pg. III, 98) (Fig. Io).

Oltre ai dispositivi socioculturali dei media di massa (cinema, televisione, fumetti e cartoon, game), nella trasposizione toninelliana fanno capolino spesso simboli, brand e icone del sistema dei consumi italiano, che la televisione, soprattutto nelle forme spettacolari veicolate da Carosello (il programma di drammatizzazione delle reclame, andato in onda dal 1957 al 1977), aveva contribuito a pubblicizzare e a rendere popolari: per esempio, tra gli insetti 
che tormentano gli ignavi compaiono vari esemplari di Vespa Piaggio, per un disguido con le aziende fornitrici (If. III, I3). Ancora, quando il poeta mantovano presenta all'Alighieri il "collega" Marco Anneo Lucano, Dante si complimenta con lui per la produzione non di un poema, ma di un digestivo (If. IV, I7). La striscia umoristica raggiunge una delle sue vette quando, in seguito ad uno scambio di battute tra Virgilio, preso dallo sconforto dopo l'ennesimo goffo incidente e pronto a lasciare la sua missione, e Dante, che lo invita a rimanere al suo posto perché sarebbe stato impossibile rimediare un'altra guida, compare dal nulla l'omino Michelin (If. XXIV, 73) (Fig. II). La stessa storia di Ulisse produce un anacronismo spiazzante e irresistibile: raccontando a Dante le sue peripezie per i mari, il prode guerriero di Itaca rivela di aver trovato, alla fine del viaggio presso le colonne d'Ercole, non ciò che si sarebbe aspettato, ma... un Club Mediterranée (If. XXVI, 77), ovvero una delle prime realtà commerciali ad aver fatto conoscere agli italiani la formula del "villaggio vacanze". Un'altra citazione, ben nota ai lettori-telespettatori degli anni Ottanta e Novanta, coinvolge l'acqua Ferrarelle, il cui slogan compare, lievemente mutato, quando a Dante è chiesto di bere l'acqua dell'Eunoè, ma Matelda gli offre come alternativa l'acqua imbottigliata, "liscia, gassata o Eunoelle" (Pg. XXXIII, I49). Un ulteriore prodotto entrato nell'immaginario di massa viene omaggiato nelle strisce finali, quando due anime pescano angeli usando come esca il "pane degli angeli", con un chiaro omaggio al brand "Pane Angeli", tuttora noto per i lieviti e gli altri prodotti utili a realizzare dolci fatti in casa (Pd. XXX, 199).

Le forme del consumo mediale, sempre più centrate sull'esperienza spettacolare, con una progressione esponenziale a partire dalla metà dell'Ottocento (Abruzzese, Mancini 2007), si manifestano nelle parole di Virgilio, che confessa a Matelda che il suo modello di età dell'oro non è il Paradiso terrestre ma Disneyland (Pg. XXVIII, I39), e di Dante, che, interrogato sempre da Matel$\mathrm{da}$, ritiene che la celebre "processione" di savi e animali alati, sia il Circo Orfei (Pg. XXIX, 139). C'è anche il riferimento a una rivista di culto dei consumi mediali italiani, la Settimana Enigmistica, allorquando Dante, al cospetto dei sibillini quesiti di Beatrice, si interroga sull'opportunità di intitolare la sua opera "La Commedia enigmistica" (Pg. XXXIII, I48).

Enti e associazioni celebri contribuiscono alla trama di rinvii, noti all'autore e ai suoi lettori. Per esempio, di fronte alla riottosità dei diavoli ribelli ad aprire le porte della città infernale di Dite, Virgilio, come un qualsiasi automobilista bisognoso di soccorso, telefona all'ACI (Automobile Club d'Italia) (If. IX, 37), l'ente addetto anche all'assistenza autostradale, già reso celebre per una telefonata cult del personaggio di Furio Zòccano nel film Bianco, rosso e verdone (198I) di Carlo Verdone. Più avanti, invece, un dannato informa Dante che la pena assegnata ai violenti contro gli altri finirà presto, perché il 
sangue in cui sono immersi (per la legge del contrappasso) sarà presto aspirato dall'Avis (la onlus che promuove le donazioni di sangue) (If. XII, 46).

Non manca un divertito a riferimento a Silvio Berlusconi, definito da Elettra "l'arcoride Silvio", per la provenienza dalla nota villa di Arcore. La donna rivela ai due pellegrini che il progetto iniziale di suo figlio Dardano, fondatore di Troia, era creare una serie di città satellite di Sparta, ma egli fu anticipato, appunto, dall'intraprendente imprenditore edile meneghino (If. IV, I9). Come si comprende bene da questa striscia, la moderata satira è indirizzata al Berlusconi imprenditore edile della fine degli anni Settanta, prima quindi della sua ascesa come fondatore dell'impero televisivo di Mediaset e del partito politico Forza Italia. Un altro significativo rimando alle vicende politiche si ha allorché un diavolo, rispondendo alle proteste di Dante sulla mancata ricostruzione di un ponte crollato oltre 1300 anni prima, afferma che è stato un cattivo affare affidarne i lavori alla cooperativa che sta ricostruendo L'Aquila (If. XXII, 69). Si tratta di un interessante aggiornamento rispetto alle precedenti versioni della Commedia di Toninelli, dove ritardi e malversazioni erano riferite al terremoto che colpì l'Irpinia nel I980 (Benucci 20I8: III).

In quanto parte essenziale della cultura nazionalpopolare, non poteva mancare il calcio, che interessa a Toninelli per le passioni eccessive che smuove e alimenta. Così, Farinata degli Uberti, nobile ghibellino, esprime il "tifo" per la sua fazione, indossando una sciarpa e sventolando un bandierone neroverde degli Ultras "Fossa dei Leoni" (If. X, 40) (Fig. I2). Nelle Malebolge, i palestrati diavoli neri organizzano un torneo di calcio, in cui ciascuna squadra infernale può assumere uno "straniero" (cioè un'anima del Paradiso o del Purgatorio) e gli stessi dannati, eccezionalmente, possono smettere di patire e seguire le gare (If. XXII, 70). Più surreale e umoristica è la terza striscia riservata al "pallone": commosso per l'abbraccio tra i concittadini mantovani Sordello e Virgilio, l'Alighieri si lascia andare ad una dura invettiva contro l'Italia in preda a tragiche lotte intestine (" $\mathrm{E}$ tu, invece, Italia, schiava delle tue divisioni interne, preda di litigi, invidie e rancori, riesci solo a suscitare un senso di nausea!"). Al Poeta risponde perentoriamente ("Ahò, se credi di essere più bravo, vieni tu a fare il commissario tecnico!") un calciatore della Nazionale, parte di un piccolo gruppo di quattro atleti tra cui si riconosce il "Divin Codino" Roberto Baggio (ad ulteriore conferma del radicamento dell'immaginario toninelliano nei decenni Ottanta e Novanta) (Pg. VI, I04). Nel tourbillon dei materiali culturali contemporanei, che puntellano ironicamente la macchina mediale di Toninelli, c'è spazio anche per qualche altra celebrità sportiva nazionale: Dante, preoccupato per la salita impervia, si lamenta con Virgilio che ciò che si prospetta davanti a loro è "roba da scalatori" e che lui non è "mica Reinhold Messner", citando uno tra i più celebri alpinisti italiani $(P g . \mathrm{X}$, I09). 


\section{INTERMEdialità, PASTICHE E PAROdia}

Questo incandescente materiale richiede un'interpretazione teorica sulle modalità con cui opera la macchina intermediale congegnata da Toninelli. Com'è noto, Genette (1997) distingue in maniera netta tra parodia e pastiche, due categorie evidentemente connesse con l'estetica e la poetica toninelliane: mentre la prima è sostanzialmente una variazione in chiave ludica di un'opera singola, il secondo consiste nell'imitazione di un insieme di testi o di un intero genere. In realtà, come fa notare Fusillo (2016: I), lo stesso Genette ammette che il suo lavoro mira a scompaginare questa dicotomia, mantenendo, invece, quella essenziale tra imitazione (il registro che accomuna parodia e pastiche) e trasformazione. È Jameson (2007) a inquadrare il pastiche come una delle forme culturali prevalenti nel postmoderno, in quanto dimensione di un'opera in cui abbondano citazioni e omaggi, più o meno superficiali, e che, pertanto, non è classificabile né come una copia né come un originale. Nell'ottica di Jameson, la parodia non sarebbe più praticabile nell'era postmoderna, perché manca un'opera unica, il cui linguaggio sia condiviso tra autore (che parodizza) e lettore. Il cuore dell'estetica postmoderna consiste in un saccheggio degli stili del passato, laddove il modernismo è l'era degli stili elevati e inarrivabili. In realtà, come fa notare Bottiroli (2006), nel postmoderno abbondano sia pastiche che parodie: il punto è capire quale delle due categorie prevale in un certo contesto socioculturale e in un certo momento storico, soprattutto perché da tale prevalenza dipende una certa interpretazione del concetto di intermedialità.

Il Dante di Toninelli costituisce un caso liminale. Da un lato, il fumetto dell'autore senese è sicuramente inquadrabile nella parodia, in quanto il suo punto di partenza è l'opera capitale della letteratura italiana, i cui riferimenti, pertanto, sono ben noti alla comunità dei lettori. Dall'altro lato, tra vignette e baloon, è possibile rintracciare simboli, icone e rinvii all'immaginario pop contemporaneo, in quantità e qualità tali da richiedere un'ulteriore esplorazione analitica - un'operazione tipicamente riconducibile all'estetica contemporanea propria del pastiche.

Arrivati a questo punto, forse occorre uno sforzo di chiarimento teorico sul concetto di intermedialità, poiché, se sicuramente l'operazione di Toninelli esibisce una natura intermediale, il senso dello stare "tra" due o più media si può pienamente cogliere solo ragionando compiutamente sullo scenario teorico in cui si inserisce l'intermedialità.

Autori come Manovich (2002), Bolter e Grusin (2002) e Henry Jenkins (2006), ragionando rispettivamente sulle logiche del remix, della rimediazione e della convergenza tecno-culturale, hanno messo in evidenza come il pastiche, essendo senza dubbio una delle forme centrali della cultura contemporanea, non possa essere ridotto a un insieme di citazioni e omaggi, in quanto 
agisce secondo spinte metamorfiche volte a riplasmare le culture, gli stili, i media del presente e del passato. In altri termini, il pastiche contempla in egual misura "continuità e differenziazione" in quanto "due macrotendenze dell'intertestualità contemporanea" (Fusillo 20I6: 5), come dimostrano anche numerosi contributi nell'area dei Remix Studies (Navas, Gallagher, Burrough 20I5, Campanelli 20II).

Ma cosa intendiamo per intermedialità? Già Marshall McLuhan (I967) aveva evidenziato come dalla collisione, fusione, mix di due media si scatenano delle energie culturali positive, generando nuove forme di interazione tra pubblici e media e rinnovando l'intero mediascape. Nel campo degli studi sull'intermedialità, arricchitosi negli ultimi anni di numerosi contributi (Mariniello 2008), una delle classificazioni maggiormente condivise è quella elaborata da Rajewski (2002), che distingue tra tre tipi di intermedialità: I) la trasposizione, per cui il contenuto di un medium viene adattato in un altro medium (es. un film tratto da un romanzo); 2) la combinazione, per cui più contenuti mediali convergono in uno stesso testo (è il caso del cinema, che sintetizza audio, immagini fisse e in movimento, fotografia, ecc.); 3 ) il riferimento, ovvero il caso in cui un'opera contiene rimandi, evocazioni, descrizioni, tematizzazioni del contenuto o delle tecniche rappresentative di un altro medium (es. descrizione scritta di una scena di un fumetto o di un film). Sintetizzando, con Frezza (2015: 53) possiamo definire l'intermedialità come "ciò che è comune fra i diversi media, un 'comune' che dunque può essere scambiato fra di loro ma non necessariamente"; in questo senso, l'intermedialità può essere interpretata come "una componente importante della transmedialità; si può dire che l'intermediale nutre e vivifica il transmediale" (Frezza 2015: 53). Tuttavia, per evitare di ingenerare confusione, i due concetti vanno opportunamente distinti: per transmedialità intendiamo infatti la qualità di quei progetti comunicativi che utilizzano in maniera integrata più media. Un esempio di progetto transmediale è la saga di Star Wars, di cui ciascun medium (cinema, fumetto, serialità televisiva, letteratura di genere) racconta alcuni tasselli che, uniti assieme, vanno a costruire l'ampiezza del transmedia storytelling (Andò e Leonzi 20I4, Kalogeras 20I4, Giovagnoli 20II, Jenkins 2003, 2006) di questo franchise.

Tornando al nostro oggetto di ricerca, si potrebbe affermare che Toninelli, in Dante, applichi due tra i modelli di intermedialità classificati da Rajewski: la trasposizione, essendo il suo fumetto un tentativo di tradurre nel linguaggio dei fumetti la Divina Commedia dantesca - con una dichiarata fedeltà all'originale -, e il riferimento, poiché la quantità di rinvii si manifesta sia nella forma del semplice omaggio (tra cinema, musica, costume, pubblicità, letteratura e così via), sia in quella della descrizione delle tecniche rappresentative (si veda la citata sequenza dei "pietroni animati"). Occorre precisare che resta 
preponderante, in questa macchina mediale, la struttura della striscia comica: ogni riferimento è finalizzato a massimizzare l'effetto ironico, comico o satirico, attraverso risorse retoriche, - come il gioco di parole, il calembour, il non-sense e altre ancora - la cui forza simbolica si arricchisce nella dimensione del paradosso spaziotemporale (i riferimenti sono quasi tutti relativi ad avvenimenti, opere e personaggi di un'epoca successiva a quella in cui il Sommo Poeta ha redatto la Commedia). D'altronde, gli omaggi e i richiami all'interno del fumetto non incidono sull'estetica dello stesso: i personaggi citati, infatti, sono sempre disegnati secondo la cifra stilistica ben identificabile dell'autore, senza intrecciare il proprio con altri stili figurativi (fatte salve poche eccezioni: le citazioni dei manga giapponesi, di Bonaventura di Sergio Tofano, di Eta Beta e dei tre porcellini disneyani). Inoltre va ricordato che, in moltissime occasioni, la citazione di Toninelli è elaborata sotto forma di richiamo testuale e non visivo.

In questo senso, possiamo dire che il fumettista senese gioca una raffinatissima partita estetico-mediale, per un verso conservando il modello nobile della parodia - come dimostra tra l'altro l'affinità di molte parti del Dante con i poemi eroicomici, esempio di parodia par excellence - e per l'altro adottando l'estetica del pastiche, sempre dentro una strategia che ha al centro la cultura visuale e le regole rappresentative della strip comica.

Ciò che è più interessante, in realtà, è il tipo di interazione stimolata da questa macchina intermediale. Come ha adeguatamente argomentato Montani (20I0), l'intermedialità è una proprietà di quelle narrazioni in grado di attivare un processo di "autenticazione", basato su un esercizio critico dell'immaginazione capace di riflettere sulla diversità dei contenuti intermediali in gioco. Dunque, nella Commedia di Toninelli lo spettatore è continuamente interpellato, sulla scorta di quella discrasia tra la piattaforma testuale di partenza (già in sé ricchissima di echi, nonché rielaborata in una vasta tradizione iconico-figurativa: Amendola, Tirino 20I6) e i tanti riferimenti all'immaginario pop: in questo spazio "tra" il poema dantesco, la trasposizione fumettistica e i riferimenti ad altri prodotti culturali - uno spazio, quindi, costitutivamente intermediale - si misura una distanza di tipo innanzitutto temporale (il paradosso di cui scrivevamo poc'anzi), ma anche spaziale (la presenza nei gironi infernali di personaggi che non potrebbero comparirvi, per esempio) e, in misura minore, estetica (per l'apparizione di elementi grafico-stilistici in conflitto con lo stile, assai riconoscibile, del tratto di Toninelli). L'immaginazione critica, dunque, è la dimensione fondamentale attraverso la quale lo spettatore è chiamato ad interpretare il gioco ironico, comico o satirico costruito su questi difformi, eterogenei ed effervescenti materiali culturali. 


\section{Conclusioni. Il Dante di Toninelli e la SERialità}

Rimane comunque consistente la vena didattica. Toninelli non dimentica mai qual è il suo pubblico: la sua Commedia utilizza tutti gli espedienti citati sicuramente per avvicinare la difficile opera ai giovani lettori. I contenuti originari del poema sono certamente semplificati, ma è una via per raggiungere e appassionare nuovi lettori. A volte la parodia riesce anche ad illuminare alcuni dei significati più profondi di questo classico che come una inesauribile miniera, parafrasando Calvino (1995), non finirà mai di dire quel che ha da dire. La pietà per le anime, l'orrore per le tremende pene, si trasformano in occasione comica; la varietà di personaggi e aneddoti diventano, per l'autore, fonte inesauribile di gag. Il congegno parodico, ovviamente, regge meglio nell'adattamento dell'Inferno, anche grazie alla fama di molti personaggi su cui è più facile costruire il gioco comico. Nel Purgatorio e nel Paradiso diventa sempre più necessario spiegare i difficili concetti teologici e le vicende storiche presentate da Dante, ecco allora che l'accompagnamento didascalico-didattico si fa più costante. Nel Paradiso, quindi, il legame tra i personaggi danteschi e quelli parodiati si fa inevitabilmente più flebile e la comicità si sposta completamente sui giochi di parole e sullo slittamento dei contesti; gli aspetti tragici, il sublime, le invettive, le rivelazioni, vengono attualizzati attraverso la banalità e la superficialità del mondo contemporaneo. Insomma in questa operazione parodica emerge il corpo a corpo che Toninelli ha dovuto ingaggiare con le fonti testuali e dal quale emergono elementi strutturali della Commedia, altrimenti in secondo piano: in primis le profonde tracce che l'opera ha lasciato nella nostra cultura, insieme con la potenza di un immaginario che ha attraversato secoli e culture diverse.

Un prodotto seriale come questo dà vita a "un movimento esploratore che sente la necessità di ritornare ai luoghi fondatori, in un viaggio che non è tanto quello della melanconia della perdita quanto il frutto della necessità di conoscere le fonti del passato per affrontare nuove sfide creative" (Balló, Pérez 2007: 219). D'altronde questo è un viaggio a cui prese parte lo stesso Dante che per la sua Commedia rivisita luoghi e forme dell'Eneide, e non solo (Bellomo 20I2). Intraprendere una narrazione a spirale, con la pretesa di emulare un testo precedente, significa anche penetrare nel suo mistero, collocandosi nella stessa situazione dell'autore del passato e partecipando alla stessa complessità testuale (Balló, Pérez 2007: 220). Questo fumetto, rivisitando un classico per proiettarsi in un futuro, mette allora in luce alcune caratteristiche della Commedia ancora poco analizzate, innanzitutto la sua "sgangherabilità" (Eco I994), ovvero la sua particolare costruzione a blocchi, in cui ogni segmento narrativo può essere smontato e ripreso come archetipo narrativo o come citazione: proprietà, questa, che la rende particolarmente adatta a riscritture 
seriali. Sicuramente il gran numero di personaggi e vicende che appaiono sono già elementi utili e di inesauribile ispirazione per una riscrittura seriale, ma è la struttura stessa a nascondere alcuni elementi propri dell'universo seriale a partire dalla gerarchizzazione dei castighi e dell'organizzazione dei territori dell'oltre mondo in funzione delle colpe o dei meriti. La periodicità dei tormenti o delle beatitudini che si ripetono eternamente e il cammino, il percorso di Dante, danno vita a un sistema capace di muoversi fra ripetizione e differenziazione, che è alla base dei processi seriali e che "fornisce allo spettatore il senso dell'attesa, della previsione, e non gli nega, in nessun caso, il piacere della sorpresa" (Balló, Pérez, 2007: I2). Toninelli cerca di essere complice dei lettori e gioca con la familiarità di personaggi e situazioni, fa leva sul piacere che danno le storie già conosciute e sui cortocircuiti che le sue innovazioni comiche riescono a creare: "alla presentazione del codice che il lettore conosce (nel nostro caso l'inferno dantesco - patrimonio comune della cultura media italiana e non solo - con i suoi elementi costanti: i cerchi infernali, il sistema del contrappasso, i personaggi celebri, ecc.), codice che crea una certa predisposizione nel lettore, segue il perturbamento di quest'ultima tramite la comparsa di un evento o contesto fuori luogo, che la logica dei possibili narrativi originali esclude a priori (...) i punti di contatto fra archetipo e derivato parodico rafforzano la carica contestatrice originaria, e ampliano gli orizzonti d'attesa del lettore del rifacimento" (Benucci 20I8: IIO).

Egli riesce a far proprio il testo dantesco con leggerezza e con rispetto, tuttavia senza eccessivi timori riverenziali, anzi con la consapevolezza che il ritorno alle origini possa dare impulso al motore creativo; così al di là del valore didattico che questo fumetto può assumere, l'opera di Toninelli nel suo attraversamento della storia culturale italiana riesce a porsi a tutti gli effetti come un classico capace di accompagnare più generazioni di lettori e di trasformare Dante, grazie alle proprietà della serialità, da sommo poeta, intoccabile, a comune personaggio, capace di accompagnarci nella quotidianità. "Mossa dall'ammirazione incondizionata per una data opera che accompagna da sempre il fumettista, la parodia preserva in definitiva l'autorità dell'originale più di quanto non la distrugga, e fa emergere prima di tutto la necessità di conoscere e ri-conoscere l'originale stesso. Attraverso un infantilismo ostentato e rivendicato, il fumettista, inventore senza colpa che si diverte a scherzare sui vizi comuni, offre il proprio omaggio al genio imperituro dello scrittore dell'Inferno" (Benucci 2018: I20). 


\section{BibLIOGRAFIA}

Abruzzese, A., I973, Forme estetiche e società di massa, Venezia, Marsilio.

-, 2007, La grande scimmia, Roma, Sossella.

Abruzzese, A., Mancini P., 2007, Sociologie della comunicazione, Roma-Bari, Laterza.

Amendola, A., Tirino M., 2016, "Il filtro di Dante. L'impronta di Gustave Doré dal cinema muto al digitale", Dante e l'arte, 3, pp. II-38.

Andò, R., Leonzi, S., 20I4, Transmedia storytelling e audience management, Roma, Armando.

Balló, J., Pérez, X., 2007, Io sono già stato qui. Fiction e ripetizione, Santa Maria Capua Vetere, Ipermedium.

Barbieri, D., I990, I linguaggi del fumetto, Milano, Bompiani.

- 2009, Breve storia della letteratura a fumetti, Roma, Carocci.

Bellomo, S., 20I2, Filologia e critica dantesca, Brescia, La Scuola.

Benucci, A., 20I8, "Dalla Commedia ai comics: costanti e varianti di critica sociale nelle riscritture fumettistiche contemporanee dell'Inferno di Dante", in Lazzarin, S., e Dutel, J. (a cura di), Dante pop. La Divina Commedia nella letteratura e nella cultura popolare contemporanea, Manziana, Vecchiarelli, pp. IO7-I2O.

Bolter, J.D., Grusin, R., I999, Remediation: Understanding New Media, Cambridge, Mit Press.

Bottiroli, G., 2006, Che cos'è la teoria della letteratura? Fondamenti e problemi, Einaudi, Torino.

Brambilla, A., 20I5, "Fare commedia della Commedia. Il Dante umoristico di Marcello Toninelli”, Fumettologica, http://www.fumettologica.it/2015/12/dante-toninellirecensione/ [ultimo accesso 20.04.20I8].

Calvino, I., 1995, Perché leggere i classici, Milano, Mondadori.

Campanelli, V., 20II, Remix It Yourself. Analisi socio-estetica delle forme comunicative del Web, Bologna, Clueb.

Colombo, F., I998, La cultura sottile. Media e industria culturale italiana dall'Ottocento ad oggi, Milano, Bompiani.

Eco, U., I994, Sei passeggiate nei boschi narrativi, Milano, Bompiani.

Frezza, G., 1987, La scrittura malinconica, Sceneggiatura e serialità nel fumetto italiano, Firenze, La Nuova Italia.

-, 2006, Effetto notte, Roma, Meltemi.

-, 2015, "Campi transmediali. Fra rimediazione e innovazione", Sociologia della Comunicazione, 50, pp. 5I-6I.

-, 2017, Nuvole mutanti. Scritture visive e immaginario dei fumetti, Milano, Meltemi.

Fusillo, M., 20I6, "Estetica del pastiche e intermedialità: «Povera piccina»", Between, IV(I2), pp. I-I8.

Genette, G., 1997, Palinsesti. La letteratura al secondo grado, Torino, Einaudi.

Giovagnoli, M., 2oII, Transmedia. Storytelling e comunicazione, Milano, Apogeo.

Giunta, C., 20I5, "Perché uno dovrebbe leggere Dante?", Internazionale, https://www. internazionale.it/weekend/2015/03/15/perche-leggere-dante-commedia [ultimo accesso 20.04.2018].

Jameson, F., 2007, Postmodernismo ovvero La logica culturale del tardo capitalismo, Roma, Fazi.

Jenkins, H., 2003, "Transmedia storytelling”, Technology Review, I3(I5), pp. 3-5.

—, 2006, Convergence Culture, New York, New York University Press.

Kalogeras, S., 20I4, Transmedia Storytelling and the New Era of Media Convergence in 
Higher Education, New York, Palgrave Macmillan.

Manovich, L., 2002, Il linguaggio dei nuovi media, Milano, Olivares.

Mariniello, S., 2008, "L'intermedialità dieci anni dopo", in De Giusti, L. (a cura di), Immagini migranti. Forme intermediali del cinema nell'era digitale, Venezia, Marsilio, pp. 2I-36.

McLuhan, M., 1967, Gli strumenti del comunicare, Milano, Il Saggiatore.

Montani, P., 2oIo, L’immaginazione intermediale, Roma-Bari, Laterza.

Morin, E., 20I7, Lo spirito del tempo, Milano, Meltemi.

Navas, E., Gallagher, O., burrough, x. (eds.), 20I5, The Routledge Companion to Remix Studies, London-New York, Routledge.

Pasut, F., 2006, "Codici miniati della Commedia a Firenze attorno al I330: questioni attributive e di cronologia", Rivista di studi danteschi, VI (2), pp. 379-409.

Pellitteri, M., 2012, Mazinga Nostalgia. Storia, valori e linguaggi della Goldrakegeneration, Roma, Coniglio.

Rajewski, I., 2002, Intermedialität, Tübingen, Francke.

Ricci, L.B., 20II, "La tradizione figurata della Commedia. Appunti per una storia", Critica del testo, XIV(2), pp. 547-579.

Salerno, V., 20I6, "Commedie' di Dante: riscritture parodiche nella letteratura illustrata contemporanea”, Between, VI(I2), pp. I-II.

Teti, M., 20I2, Generazione Goldrake, Milano, Mimesis.

Toninelli, M., 2015, Dante. La Divina Commedia a fumetti, Brescia, Shockdom.

\section{RINGRAZIAMENTI}

Gli autori ringraziano sentitamente, per la collaborazione offerta, la casa editrice Shockdom. 

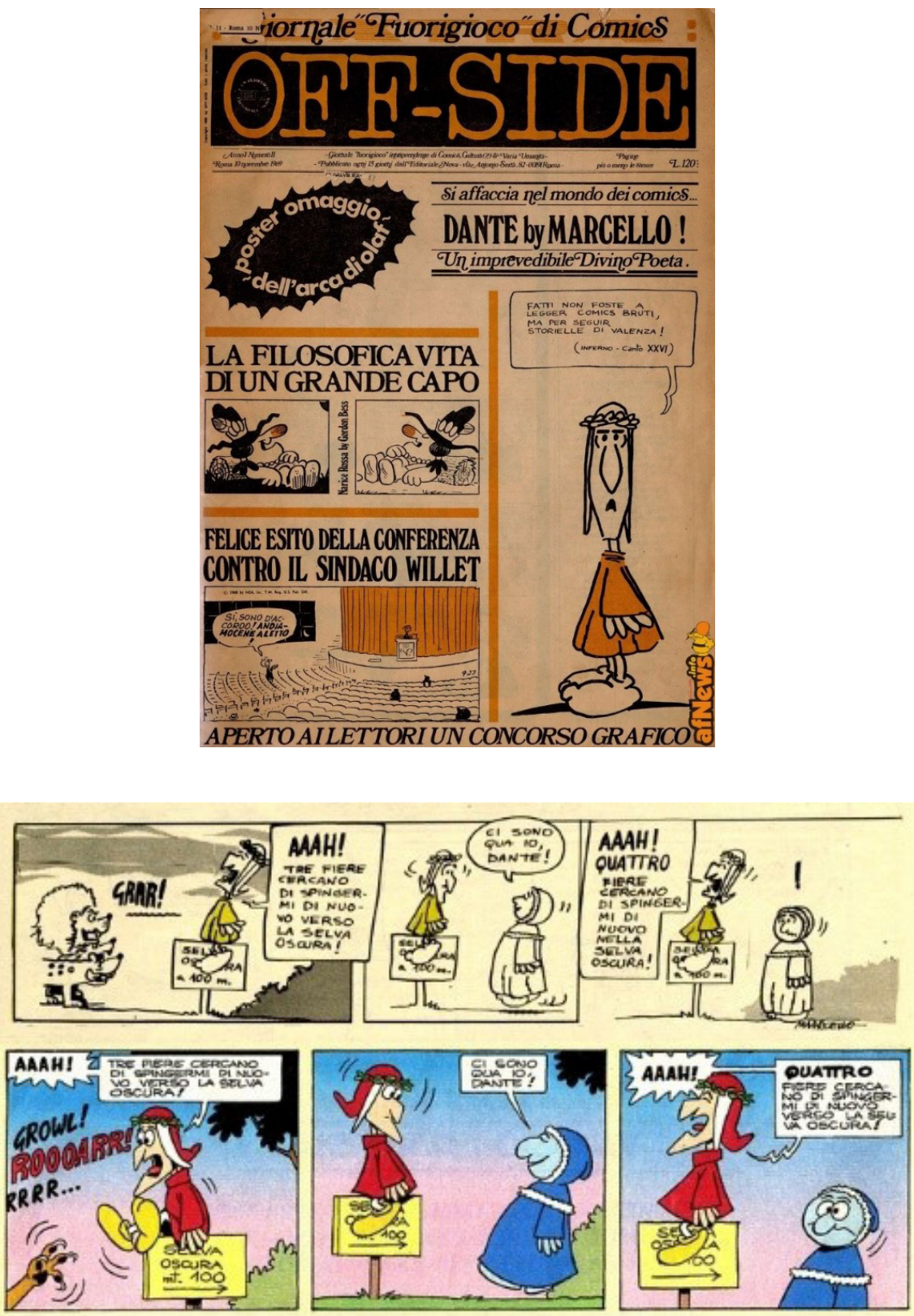

Figura I. Copertina Offside, numero II (Io novembre 1969).

Figura 2. Confronto tra la versione pubblicata su Offside (in alto) e quella pubblicata da $I l$ Giornalino (in basso). 

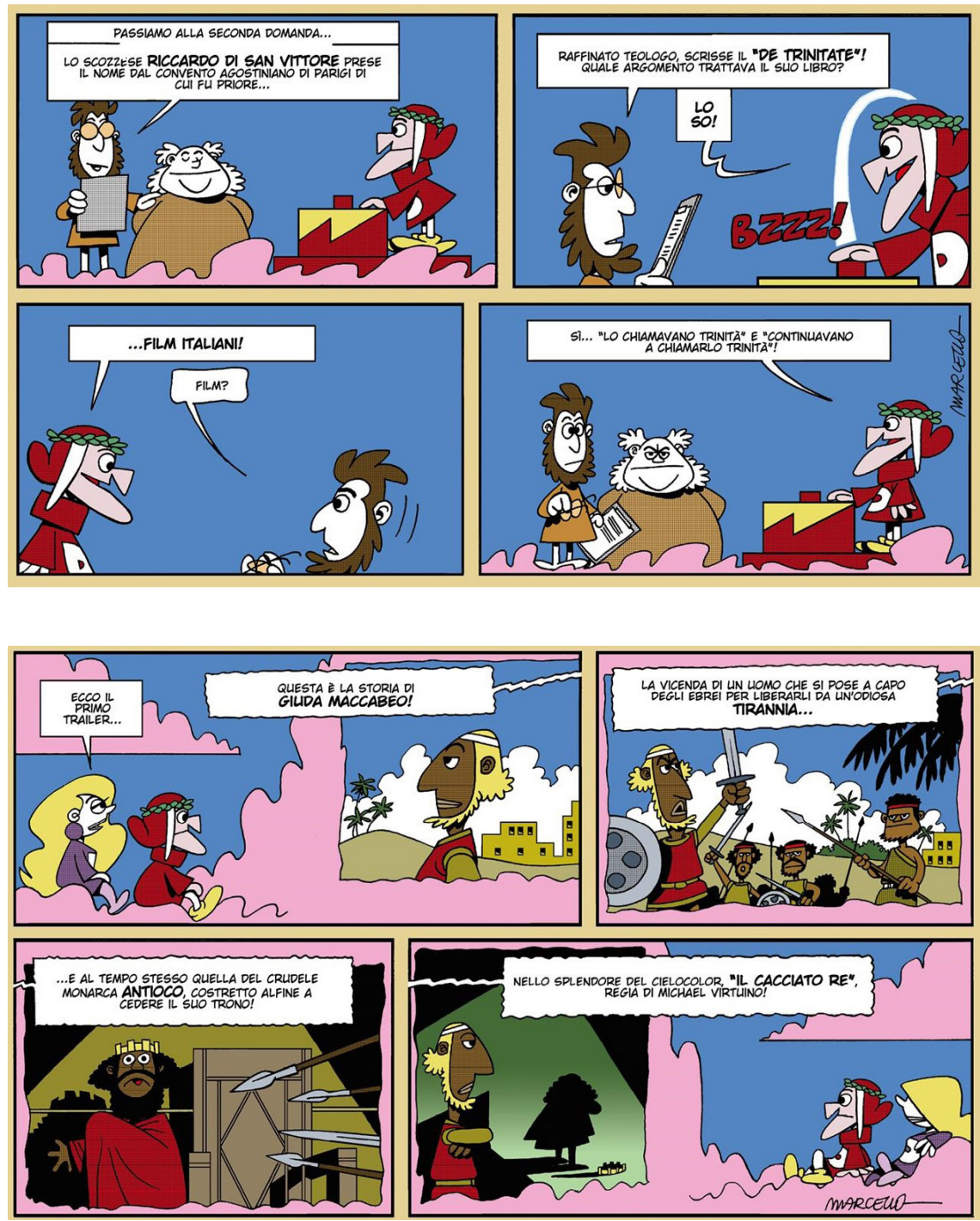

Figura 3. Citazione di Lo chiamavano Trinità e ...continuavano a chiamarlo Trinità (Dante. La Divina Commedia a fumetti, 2015, p. 163).

Figura 4. Il trailer del film Il cacciato re di Michael Virtuino (da Il cacciatore, 1978, di Michael Cimino) (Dante. La Divina Commedia a fumetti, 2015, p. I80). 

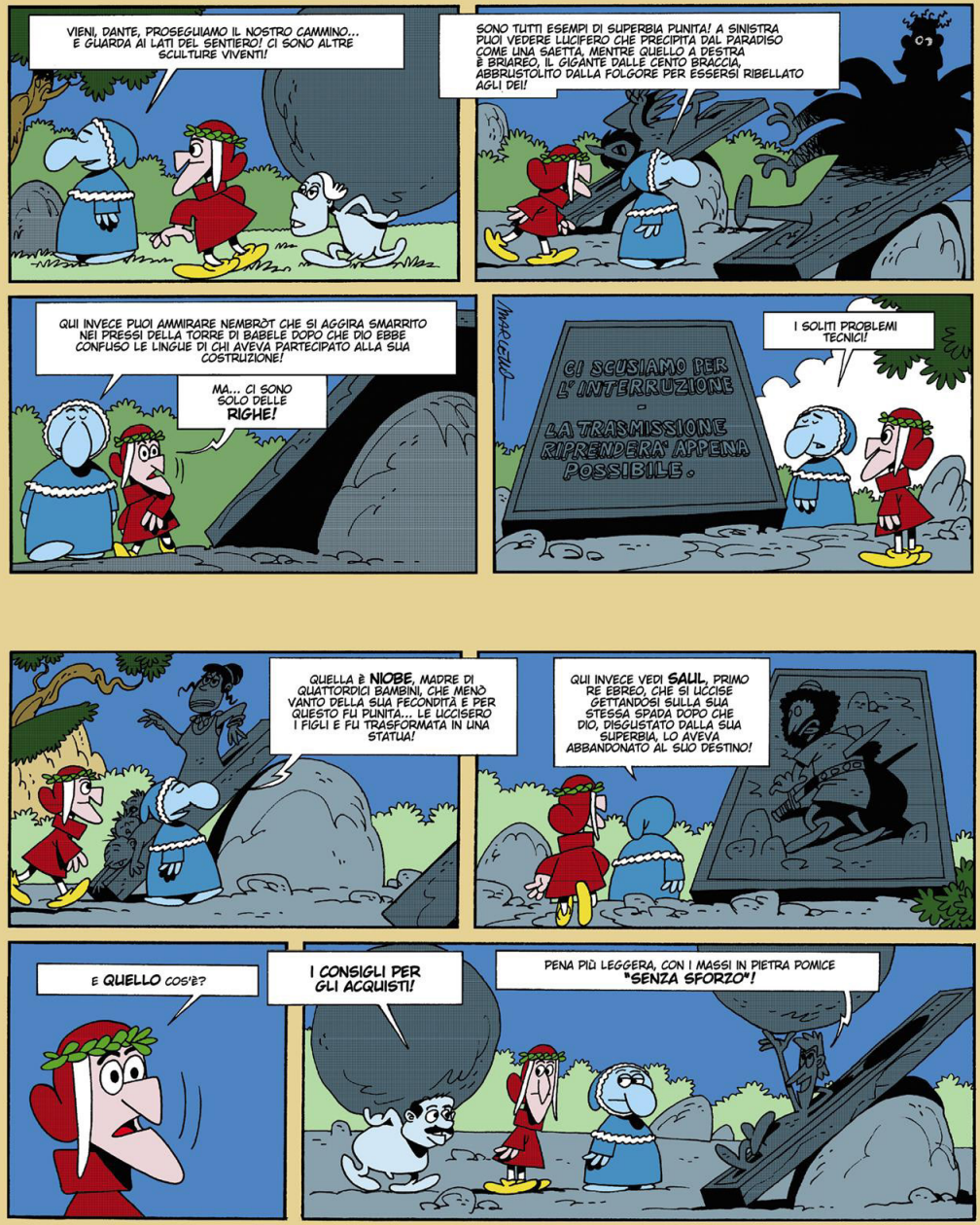

112

Figura sa. Organizzazione delle sculture viventi come un flusso di immagini televisive (Dante. La Divina Commedia a fumetti, 20I5, pp. II2-II3). 


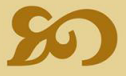

Ofolle Aragne, sivedea io te già mezza ragna, trista in su li stracci

delloperachemal pertesifé.

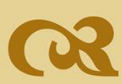

(Purgatorio, CantoXII, versi 43-45)
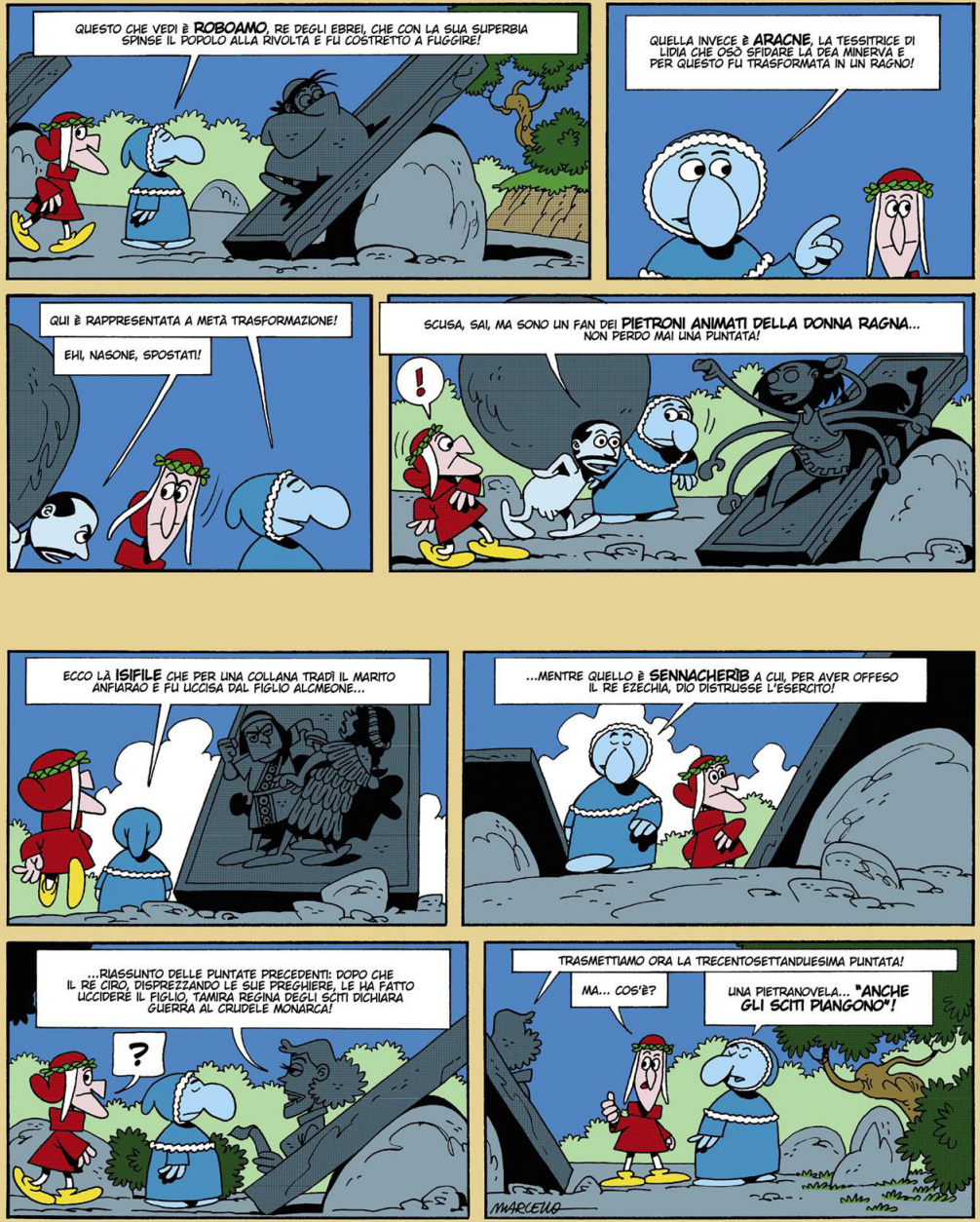

113

Figura 5 b. Organizzazione delle sculture viventi come un flusso di immagini televisive (Dante. La Divina Commedia a fumetti, 20I5, pp. II2-II3). 


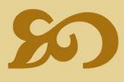

"(...) Io fui delli agni della santa greggia

che Domenico mena per cammino

u'ben simpingua se non si vaneggia. (...)"

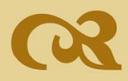

(Paradiso, CantoX, versi 94-96)
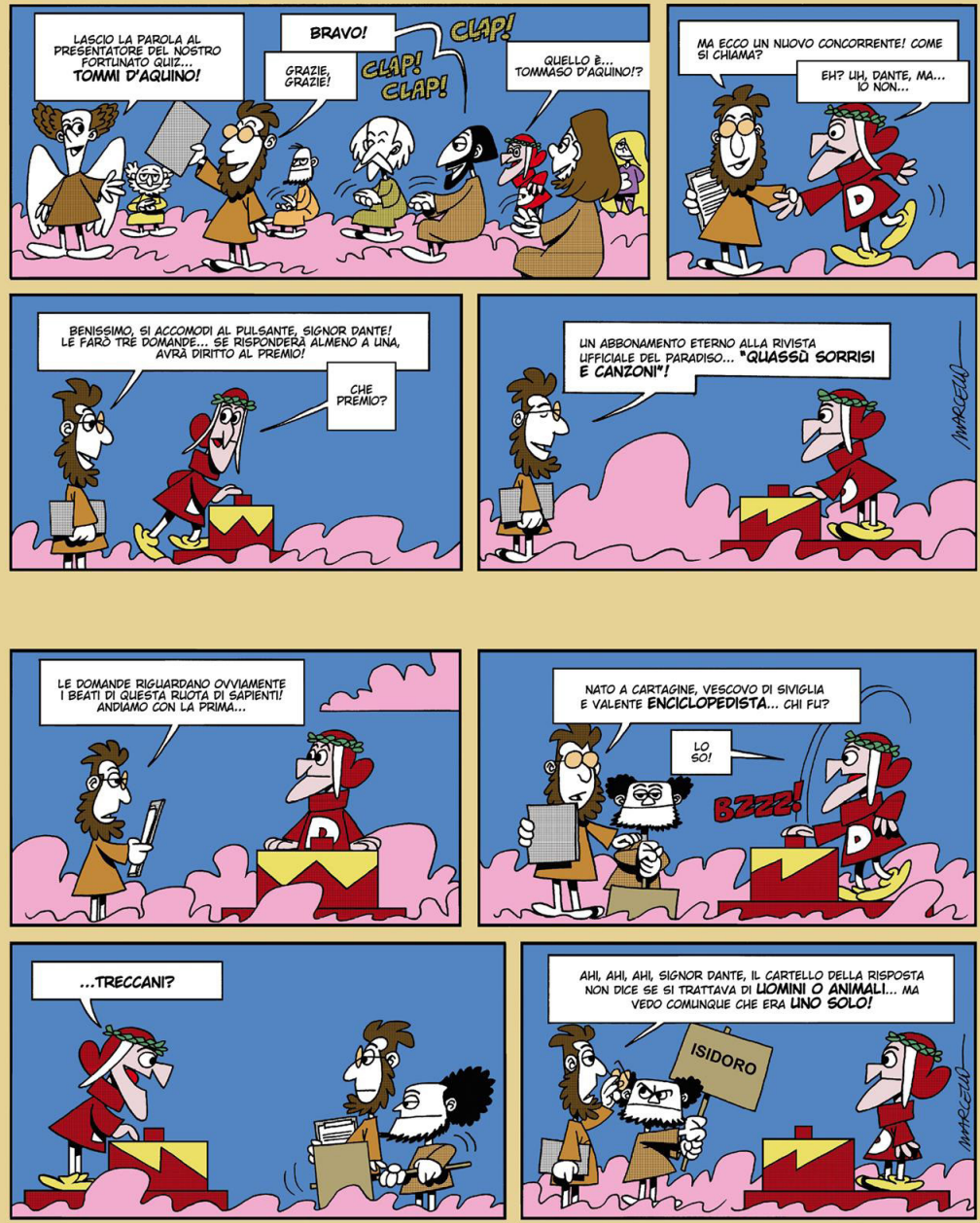

162

Figura 6. San Tommaso d'Aquino disegnato come una sorta di Mike Bongiorno del Paradiso (Dante. La Divina Commedia a fumetti, 2015, p. 162). 

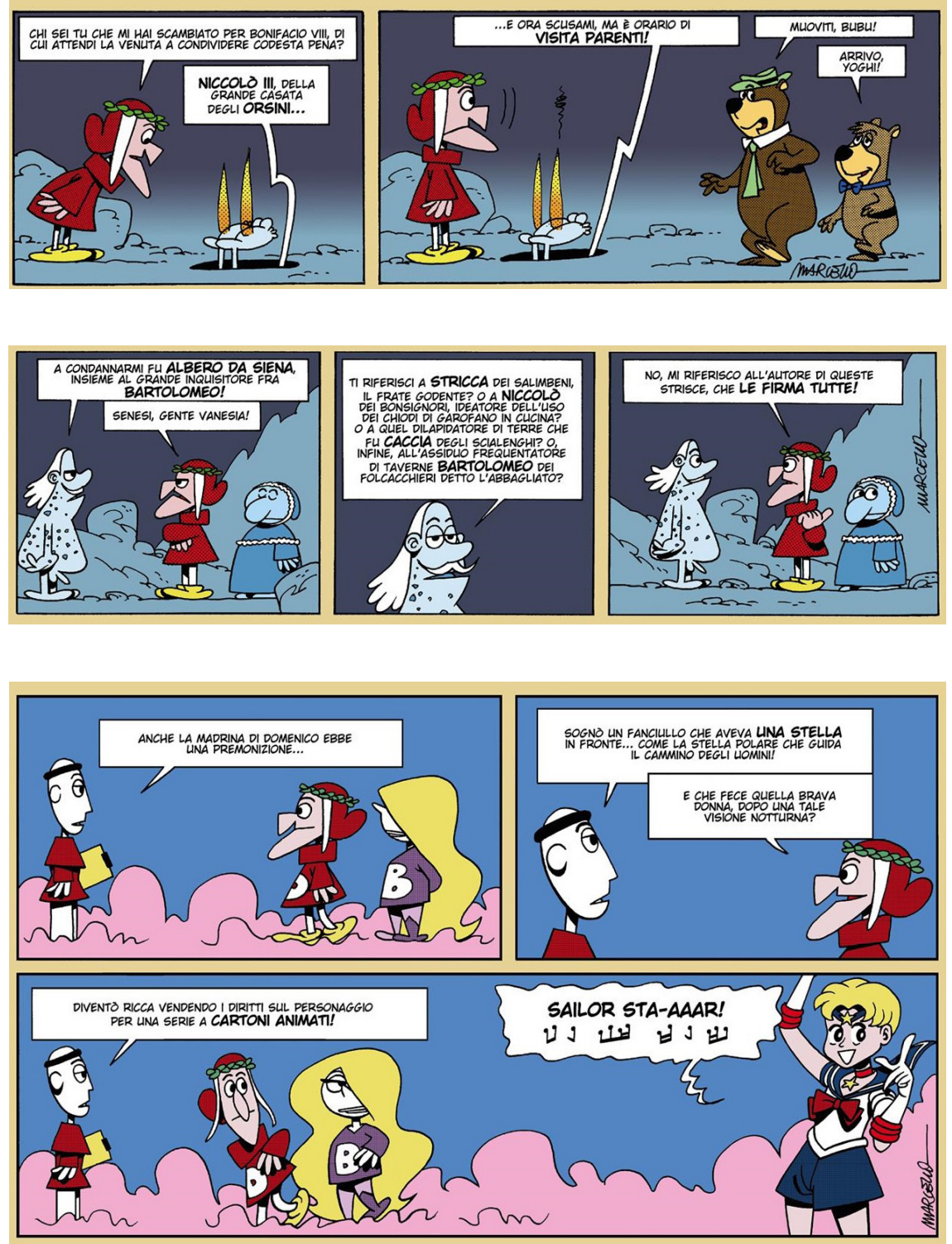

Figura 7. Gli orsi Yoghi e Bubu, personaggi animati creati dallo studio Hanna-Barbera, in visita come "parenti" a Niccolò III degli Orsini (Dante. La Divina Commedia a fumetti, 20I5, p. 63). Figura 8. Un esempio di metafumetto (Dante. La Divina Commedia a fumetti, 2015, p. 82).

Figura 9. Citazione del manga Sailor Moon (Dante. La Divina Commedia a fumetti, 20I5, p. 169). 

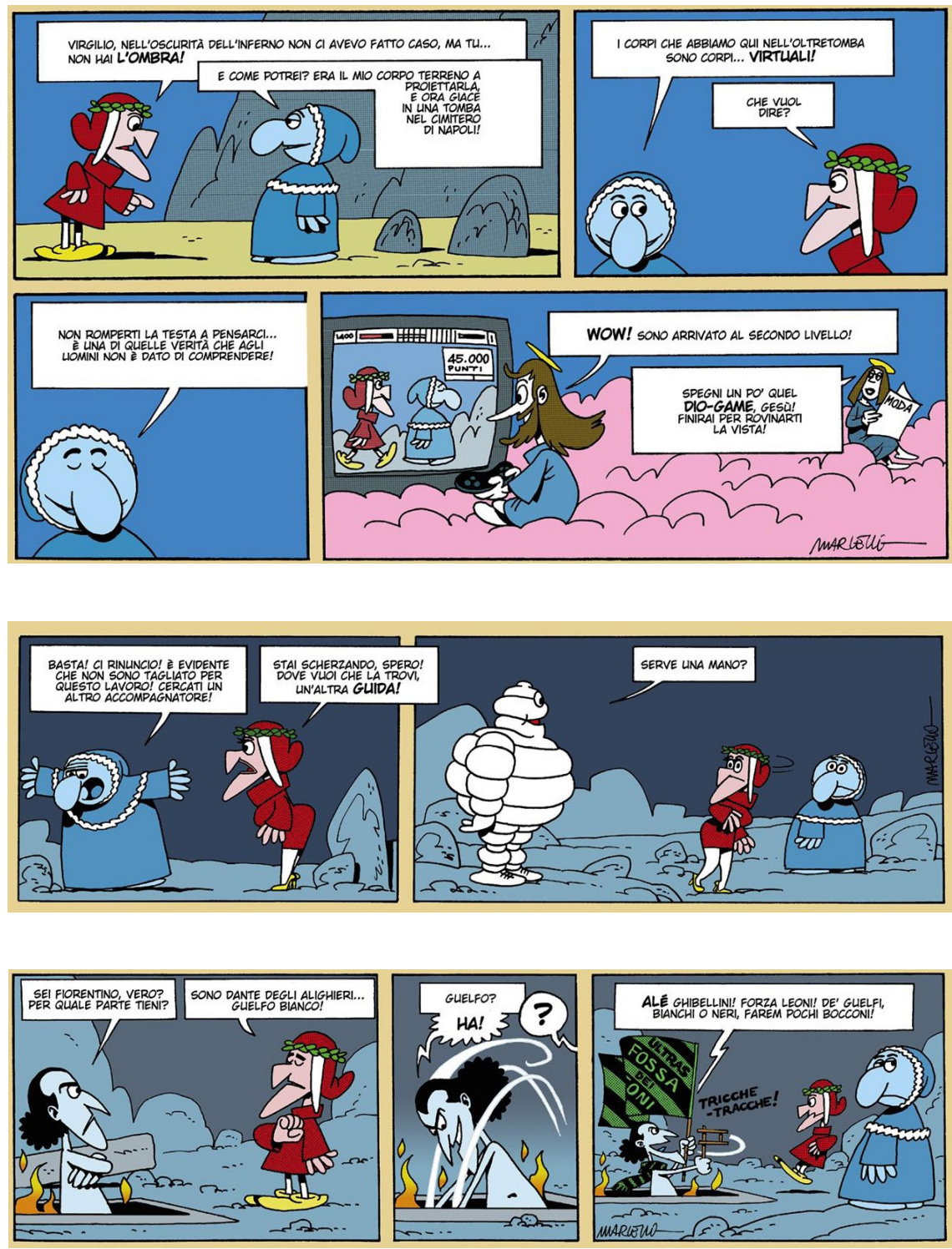

Figura ro. Gesù gioca a Dio-Game (Dante. La Divina Commedia a fumetti, 2015, p. 98).

Figura II. L'apparizione dell'omino Michelin (Dante. La Divina Commedia a fumetti, 2015, p. 73). Figura I2. Farinata degli Uberti, capo "ultras" dei ghibellini (Dante. La Divina Commedia a fumetti, 20I5, p. 40). 
